\title{
The coherence of memories for trauma: Evidence from posttraumatic stress disorder
}

\author{
David C. Rubin * \\ Department of Psychology and Neuroscience, Duke University, Box 90086, Durham, North Carolina 27708-0086, United States
}

\section{A R T I C L E I N F O}

\section{Article history:}

Received 12 December 2009

Available online 21 April 2010

\section{Keywords:}

Autobiographical memory

Narrative coherence

Posttraumatic stress disorder

\begin{abstract}
A B S T R A C T
Participants with posttraumatic stress disorder (PTSD) and participants with a trauma but without PTSD wrote narratives of their trauma and, for comparison, of the most-important and the happiest events that occurred within a year of their trauma. They then rated these three events on coherence. Based on participants' self-ratings and on naïve-observer scorings of the participants' narratives, memories of traumas were not more incoherent than the comparison memories in participants in general or in participants with PTSD. This study comprehensively assesses narrative coherence using a full two (PTSD or not) by two (traumatic event or not) design. The results are counter to most prevalent theoretical views of memory for trauma.
\end{abstract}

(c) 2010 Elsevier Inc. All rights reserved.

\section{Introduction}

Fragmented, incomplete, difficult to access, incoherent memories of a traumatic event have been an assumed normal reaction to extreme stress as well as a symptom of posttraumatic stress disorder (PTSD) (Brewin \& Holmes, 2003; Dalgleish, 2004; Horowitz, 1976). Here we add to the growing evidence that this view of traumatic memories and their role in PTSD lacks empirical support (e.g., Geraerts et al., 2007; Gray \& Lombardo, 2001; McNally, 2003a, 2003b; Porter \& Birt, 2001; Shobe \& Kihlstrom, 1997; Zoellner \& Bittenger, 2004). We begin by briefly reviewing the history, importance, and widespread nature of the hypothesis that memories of traumatic events are incoherent and are especially incoherent in people with PTSD. Then we review the substantial evidence that there is little or no data to support this hypothesis before providing a test.

\subsection{The assumed incoherence of trauma memories}

Horowitz' (1976) theory of stress response syndromes, which became the basis for the diagnostic category of PTSD (see Berntsen, Rubin, \& Bohni, 2008 for a review), holds that incomplete initial processing of a traumatic event along with analytic defense mechanisms produced an incomplete, incoherent memory of the traumatic event. A need to complete processing the memory (i.e., a Zeigarnik effect, Horowitz, 1976, pp. 92) keeps the memory active and often intrusive while attempts are made to integrate it into a more coherent form. This theoretical view has been extended by many current theories of PTSD. It holds that a traumatic event causes disruptive effects on the encoding and integration of the traumatic event in memory through mechanisms such as peritraumatic dissociation, thus leading to impaired voluntary access to a coherent trauma memory (e.g., van der Kolk \& Fisler, 1995). For instance, Ehlers and Clark (2000) note that "patients often have difficulty in intentionally retrieving a complete memory of the traumatic event. Their intentional recall is fragmented and poorly organized, details may be missing and they have difficulties recalling the exact temporal order of the events" (p. 324).

\footnotetext{
* Fax: +1 9196605726 .

E-mail address: david.rubin@duke.edu
} 
Brewin, Dalgleish, and Joseph's (1996) dual representation theory assumes conscious, verbally accessible "memories of trauma will tend to be vague, poorly organized and show gaps and discontinuities" (Brewin, 2001, pp. 161; for more detailed reviews see Berntsen, 2009; Brewin \& Holmes, 2003; Dalgleish, 2004; Rubin, Boals, \& Berntsen, 2008). Thus, the incoherence of the trauma memory has long been central to the understanding of memory for traumatic events in general and of PTSD in particular. Moreover, it remains as one of the 17 DSM-IV-TR symptoms of PTSD, the C3 symptom or "inability to recall an important aspect of the trauma" (American Psychiatric Association, 2000, pp. 467-468). Please note that these claims are about willful, voluntary recall of the trauma, not about involuntary recall including flashbacks.

Two hypotheses follow from this view of the effects of trauma on memory. The main-effects hypothesis is based on the continuity of human reactions to trauma, which reaches psychopathology in only a minority of cases. Given traumas serious enough to allow a diagnosis of PTSD and a group of participants varying in PTSD symptom severity, trauma memories should be more incoherent than comparison memories. The interaction hypothesis assumes that the degree of incoherence is diagnostic of PTSD. It holds that is that trauma memories should be especially more incoherent than comparison memories in participants with PTSD. To test these hypotheses, the most useful evidence would be the comprehensive measurement of the coherence of memories of both traumatic and control events in people with and without PTSD. In spite of being pervasive, theoretically important, and part of the official PTSD diagnosis, we know of no such study of coherence. In general, there is surprisingly little support for the hypothesis of incoherence of trauma memories in people with PTSD, or even for a simple increase in incoherence of trauma memories in all people (O'Kearney \& Perrott, 2006). We review the existing literature in three sections: coherence of the trauma in the life story, self-ratings of coherence internal to the memory, and neutral observer ratings of coherence internal to the memory.

\subsection{Coherence of the trauma in the life story}

The first approach is to ask whether the narrative of the traumatic event fits coherently into the ongoing life story of the individual (Ehlers \& Clark, 2000). Berntsen and Rubin (2006b) devised the Centrality of Event Scale (CES) to investigate this aspect of narrative coherence. It contains items such as, "I feel that this event has become part of my identity; I feel that this event has become a central part of my life story; this event was a turning point in my life." In several studies involving undergraduates, a representative sample of the Danish population, and older Danish adults who lived through the German occupation of Denmark, the scale and subsets of items from the CES have correlated with PTSD symptoms in the .35-.55 range (Berntsen \& Rubin, 2006a, 2006b, 2007, 2008; Rubin et al., 2008). Thus, in this sense of coherence, traumatic events are substantially more, rather than less, integrated and coherent to the life story in people with PTSD or PTSD symptoms. Although the correlation is in the wrong direction for the main-effects hypothesis, no test of the interaction hypothesis exists because comparison memories have not been rated in samples including participants with and without PTSD.

\subsection{Self-ratings of coherence internal to the memory}

Here too, the data do not provide support for the view that traumatic events are recalled less coherently, especially in people with PTSD; there are either no differences or statistically significant differences in the wrong direction for the incoherence view. Rubin, Feldman, and Beckham (2003) tested 50 veterans with PTSD who each recalled a memory from the 2 years before service, a non-combat service related memory, a combat memory, and the memory of the event that had most often come as an intrusive memory. There was a significant effect on how coherent the memory was rated across these four classes of memories with the combat and intrusive memories being rated as more coherent. There was no effect on whether the four classes of memories were rated as coming more in pieces. These findings are counter to the main-effects hypothesis. Berntsen, Willert, and Rubin (2003) asked 113 students about the trauma that bothered them the most. There was no difference in coherence between those with and without PTSD symptoms. Rubin, Boals, and Klein (2010, Experiment 2) asked 120 undergraduates with either high (40-73) or low (17-22) scores on the Posttraumatic Stress Disorder Check List (PCL, Weathers, Litz, Huska, \& Keane, 1994) about the coherence of their memory for their most stressful or traumatic experience and found no significant differences.

Berliner, Hyman, Thomas, and Fitzgerald (2003) found that memories of verified traumas in children differed from memories of positive events, being lower on a combined scale of sensory detail and coherence items but not on a scale composed of temporal and spatial items related to what happened before and after the event and where people and things were. Thus some aspects of coherence seemed to be affected but not others. There was no correlation of either of these scales with a trauma symptom checklist. Byrne, Hyman, and Scott (2001) found that memories for traumas and memories of negative events differed from memories of positive events but not from each other on one measure related to coherence: the memory for events occurring before the event in question. However, this effect did not hold for other measures of coherence such as the events occurring after the event in question or the order of the event. There were no clear patterns in the correlations of memory ratings of the traumatic event and the measures of PTSD symptom severity, disphoria, and dissociation use in the current manuscript. However, the measure of the memory for events occurring before the event in question for the traumatic memory did correlate 30 with another measure of symptom severity. Thus, these two studies offer at best mixed support for the main-effects and the interaction hypotheses.

Although none of these studies has the complete 2 by 2 design required to test the hypothesis that people with PTSD have less coherent trauma memories than people without PTSD (or with lower levels of symptom severity), varying either the 
symptom of the participants or the severity of the event does not produce results consistent with the idea that memories of traumatic events are less coherent in people with PTSD. Other evidence on the self-rating of coherence comes from the DSMIV-TR C3 symptom, the inability to recall an important aspect of the trauma. This symptom is an outlier, in that it does not correlate in expected ways with the other symptoms of diagnosis (Foa, Riggs, \& Gershuny, 1995; Rubin, Berntsen, \& Bohni, 2008, Table 4).

One study does have the relevant 2 by 2 design outlined earlier but did not have participants with a PTSD diagnosis. Rubin et al. (2008) had 115 undergraduates rate how coherent their memories were and whether they were fragmented. The undergraduates rated memories of their three most-stressful events, three most-positive events, their seven most-important events and 15 memories cued by words. ANOVAs comparing the most-stressful events to each of the other three kinds of comparison memories showed no effects of the PCL score and no interactions, but the most-stressful memories were more coherent than the word-cued and most-important memories and less fragmented than the word-cued memories. In general, for all of the studies using self-ratings of the internal coherence of the memory, traumatic memories in people with PTSD are not less coherent than non-traumatic memories in people with PTSD or traumatic memories in people without PTSD. Any statistically significant differences observed are in the direction opposite to the idea that traumatic memories in people with PTSD are less coherent.

\subsection{Neutral observer ratings of coherence internal to the memory}

Porter and Birt (2001) compared 306 undergraduates' narratives of their most traumatic and most-positive events and found no difference in coherence. Peace, Porter, and ten Brinke (2008) scored narratives from 44 women who reported on their sexual traumas, non-sexual traumas, and positive events. The non-sexual traumas were more coherent than the positive events, with the sexual traumas in the middle. These studies provide no support for the main-effects hypothesis. There is no study with proper controls and a good measures of coherence to test the interaction hypothesis. The closest we have is Gray and Lombardo (2001) who replicated and extended a study by Amir, Stafford, Freshman, and Foa (1998). These studies used the Flesch Reading Scale (Flesch, 1948), a mechanical measure of reading level based on the number of words per sentence and syllables per word, which was not developed as a measure of coherence. Both of these studies showed that the trauma narratives of participants with, as opposed to without, PTSD had higher Flesch Reading Scale values, which were assumed to be less coherent. Gray and Lombardo, however, also included control narratives and these showed similar effects, indicating that the results were not restricted to memories of trauma. Moreover, differences in writing skills and cognitive abilities between participants with and without PTSD could account for the main effect of PTSD on coherence. Thus, there is a clear need for control memories, but they are lacking in most other studies (e.g., Halligan, Micheal, Clark, \& Ehlers, 2003; Harvey \& Bryant, 1999; Jones, Harvey, \& Brewin, 2007; Murray, Ehlers, \& Mayou, 2002).

Ignoring the lack of proper controls, the differences in coherence rated by a neutral observer are small and do not reach the level of incoherence needed as a mechanism to produce PTSD. For instance, Jones et al. (2007) found significant differences between people with and without PTSD for memories of their trauma on their measure of global coherence. However, there was no evidence that the trauma memories of participants with PTSD were fragmented. Jones et al.'s scale ranged from $0=$ extremely coherent to $10=$ extremely incoherent with a mean rating over all times of the PTSD patients' trauma memories of 2.02. This value is far from the level one would need to use fragmentation as an explanatory mechanism. Moreover, all their participants were victims of road traffic accidents and half had traumatic brain injuries. Thus some of the lack of extremely coherent narratives (and the improvement noted over time) might be due to subclinical or observed brain injury.

Thus we can find in the literature no evidence for the main-effects or interaction hypotheses. We therefore design our study as a test of these hypotheses.

\section{Methods}

\subsection{Design}

Here we do the PTSD versus no-PTSD participants by memory of traumatic versus control events design needed to test the hypothesis that traumatic memories in people with PTSD are less coherent. We use self-report measures of coherence (expanded from Rubin et al., 2008), neutral judges' ratings of the coherence of the narratives describing the events (including a version of the scale used by Jones et al., 2007). For completeness, computer-scoring measures of narratives that have been used as measures of coherence and other properties in the literature on stressful and traumatic memory (including the Flesch Reading Scale and measures from the Linguistic Inquiry and Word Count, LIWC, Pennebaker, Booth, \& Francis, 2007).

The choice of the comparison memories is an important design decision, especially for the main-effects hypothesis. The traumatic and two comparison memories were as follows: (1) "The most stressful or traumatic event in your life that now bothers you the most, with the restriction that it occurred 1 month or longer ago." (2) "The most-positive memory that occurred within 1 year before or after the most-stressful event you listed." (3) "The most-important event that occurred within 1 year before or after the most-stressful event you listed." The 1-month restriction on the traumatic event was to ensure that it would meet the requirements for a PTSD diagnosis. The 1-year restriction on the comparison events was to ensure a similar life period for all events to control for a general loss of information over time. All events were dated to ensure the criteria 
were met. The most-positive event was included to provide a comparison event that differed mainly on valence from the traumatic event. The most-important event was included to provide a less emotional comparison event that was comparable in overall importance to the traumatic event. Thus, the two control memories were chosen to provide as close and fair a comparison as possible, both theoretically and empirically as based on past work with this population. If less noteworthy control memories were chosen, then the traumatic memories would have been more coherent than the control memories, offering stronger support for our claims at a cost of a stricter, more precise comparison (Rubin et al., 2008, Table 5).

\subsection{Materials}

The Beck Depression Index (BDI-II: Beck, Steer, \& Brown, 1996) is probably the most widely used test of depressive symptoms. The Centrality of Event Scale (CES: Berntsen \& Rubin, 2006a, Berntsen \& Rubin, 2007) measures the extent to which a traumatic memory forms a central component of personal identity, a turning point in the life story and a reference point for everyday inferences. The short form of the CES, which we used, consists of seven items. The Clinician Administered PTSD Scale (CAPS: Blake et al., 1995) is a clinical structured interview that is one standard way of formally diagnosing PTSD. The Dissociative Experience Scale (DES: Bernstein \& Putnam, 1986; Carlson \& Putnam, 1993) is a widely used measure of dissociation. The Posttraumatic Stress Disorder Checklist for specific events (PCL: Weathers et al., 1994), measures ratings to the 17 official symptoms of PTSD.

The Autobiographical Memory Questionnaire (AMQ, Rubin, Schrauf, \& Greenberg, 2003, 2004) is a series of questions asking about processes involved in remembering an event. For this study, we had one question about coherence in the life story (The event in my memory is a central part of my life story.) and six questions about coherence internal to the event. These six questions were: (1) it comes to me in words or in pictures as a coherent story or episode and not as an isolated fact, observation, or scene; (2) my memory comes to me in pieces with missing bits (reverse scored); (3) while remembering the event, I know the setting where it occurred; (4) while remembering the event, I know the location of actions within the event; (5) the order of the events before and after the event is clear; and (6) the order of the actions within the event is clear. The questions were rated on seven point scales from 'not at all' to either 'completely' or 'as if it were happening now.' As these individual measures of coherence correlated with each other ( $\alpha$ 's for the important, positive, and stressful memory $=.83, .83$, and .81 , respectively), we averaged these six scales to provide a more reliable index of self-rated coherence. We also report on the valence of the memories as a manipulation check.

\subsection{Participants}

Undergraduates were recruited either from signs on campus or from their responses on a web based prescreen. In order to ensure that our comparisons would be among trauma memories that met the current diagnostic criteria, participants were included only if a licensed or supervised, experienced, Master's level clinician judged that their trauma met the A trauma criterion of the DSM-IV-TR. All participants in the PTSD group also met the remaining DSM-IV-TR criterion for current PTSD, which was assessed by a clinician using an individually administered CAPS. There were 15 Duke University undergraduates between the ages of 18 and 22 in both the PTSD and the non-PTSD groups. As a check on the screening, we note that there was no overlap in PTSD symptom severity in the two groups measured near the end of the study; the minimum PCL score in the PTSD group was 46, the maximum PCL score in the non-PTSD group was 32.

\subsection{Procedure}

The experimental procedure was conducted within a few days of the clinical diagnosis. Participants were tested individually. They began by nominating and providing a one-line description of three memories. The participants were then given a separate page to describe each event. The order of events was randomized with the restriction that the traumatic event could not be last in order to minimize any lingering negative thoughts beyond the experimental session. An AMQ and a seven-item CES followed the one-page description each event. The CES usually measures how central a stressful event is to an individual's life. However, here we used it to measure each of the three events described. The session ended with the PCL, BDI-II, and DES.

\subsection{Rating the coherence of the written narratives}

The concept of a traumatic memory being incomplete, fragmented, or incoherent is fairly clear. However, it is more difficult to define the full range of coherence because the concept of coherence has many possible meanings. We therefore included three measures of coherence, one taken from the literature and the other two chosen to capture different aspects of coherence that would be relevant to the concept of an incoherent trauma memory. For the first, we asked raters questions about the global coherence of the text, which we averaged into one measure comprehension. These questions were intended to tap the kind of ability tested in the neuropsychological assessment of narrative comprehension (Greenberg \& Rubin, 2003). They are more abstract and more of a test of general narrative skills than of the standard measures narrative coherence as would be scored by a high school writing teacher. We chose them because of their utility in neuropsychology and as a contrast to the more detailed level provided by the coding scheme from developmental psychology, which we describe shortly. 
The questions were as follows. Do you understand what happened, that is, are the ideas that the author chose to describe clear? Do you understand more about who the narrator is, how they deal with the world, and how they must have felt? Does the text evoke an emotional reaction in you and/or empathy for the narrator? The questions were rated on a 7-point scale of: 1 , not at all; 2 , not really; 3 , a little bit; 4 , a moderate amount; 5 , yes, quite a bit; 6 , yes, very much so; and 7, yes, as much as any text of about this length could. The original disorganization question (Halligan et al., 2003) on which Jones et al. (2007) based their scale was "Overall, the text is: $0=$ not at all disorganized to $10=$ extremely disorganized." This was difficult for the scorers to use and they changed it to the following: How much of the text is disorganized - that is, how much of the writing does not add to the development of the narrative and our understanding of the narrator. This question was rated on a scale of: 1 , none; 4 , half; and 7, almost all because our raters found it easier than the original 11-point scale.

To address the more technical issues of coherence, we used the Narrative Coherence Coding Scheme (NACCS: Baker-Ward et al., 2007; Morris, Baker-Ward, \& Bauer, 2009). The NACCS was developed to provide a standard measure of the narrative coherence of essays that could be used across laboratories. The NACCS has three components, context, temporal ordering, and theme, which we combined to an average. The context rating is on the degree to which the narrator provides enough information to locate the event in space and time. The temporal ordering rating is on the degree to which the narrator provides enough information for the actions in the event to be placed on a time line. The theme rating is on the degree to which the narrator substantially develops the theme using causal linkages, interpretations, elaborations and a resolution that links this event to other autobiographical experiences or self-concept or identity and brings closure. Each is rated on a 4-point scale from 0 to 3 with specific criteria needed for each level. We did not use measures that counted the number of repetitions, disorganized thought, and unfinished thoughts as did Foa, Molnar, and Cashman (1995) for their oral sample because of the low frequency of these in our written sample.

Three paid undergraduate raters scored the typed event description of five participants who did not meet the inclusion criteria for the study. Based on this scoring, they changed the wording of the initial formulation of the rating scales to the ones reported here, which were clearer and easier to use for these data. Then they were given the three typed event descriptions of each of the 30 participants who were included in the study. These 90 essays were listed in random order, so that the raters did not know which essays came from the same participants, nor the group of the participant. The average of the three ratings was used in calculations. Cronbach's $\alpha$, calculated by correlating the three raters' scores on the 90 essays, for the comprehension, disorganization, and NACCS ratings were .61, .30, and .74, respectively. To provide a fair test of the idea that trauma memories are incoherent in people with PTSD, the ratings scales had to be defined to span the full range of coherence. This means that the ratings given were often clustered at one end of the scale, as will be shown, reducing the range of the ratings and the reliabilities that could be obtained. The .30 reliability for the disorganization is low in part because of a floor effect, as will be shown in Table 1. We retain this measure because it is a variant of one used in the literature and it will be the only measure to show an effect consistent with the main-effects hypothesis, which I wish to reject.

\subsection{Computer scoring of the texts}

The Flesch Reading Scale (Flesch, 1948) has been used as a measure of coherence in the PTSD literature, but it is actually a measure of reading level. It is calculated from the number of words per sentence and the number of syllables per word, rather than any measure of coherence. Higher numbers represent easier to read passages. We also included the main categories from the Linguistic Inquiry and Word Count (LIWC, Pennebaker et al., 2007) to provide a more comprehensive descrip-

Table 1

Measures of narrative coherence and valance.

\begin{tabular}{|c|c|c|c|c|c|c|c|c|c|c|c|c|}
\hline \multirow[t]{3}{*}{ Measures } & \multicolumn{6}{|c|}{ Means } & \multicolumn{6}{|l|}{ ANOVAs } \\
\hline & \multicolumn{2}{|c|}{ Important PTSD } & \multicolumn{2}{|c|}{ Positive PTSD } & \multicolumn{2}{|c|}{ Traumatic PTSD } & \multicolumn{2}{|l|}{ PTSD } & \multicolumn{2}{|c|}{ Memory type } & \multicolumn{2}{|c|}{ Interaction } \\
\hline & No & Yes & No & Yes & No & Yes & $F(1,28)$ & $\left(\eta^{2}\right)$ & $F(2,28)$ & $\left(\eta^{2}\right)$ & $F(2,28)$ & $\left(\eta^{2}\right)$ \\
\hline \multicolumn{13}{|c|}{ Measures from raters } \\
\hline Comprehension & 5.18 & 4.99 & 5.28 & 5.10 & 5.35 & 5.30 & 0.58 & $(.01)$ & 2.09 & $(.03)$ & 0.24 & $(.00)$ \\
\hline Disorganization & 1.58 & 1.98 & 1.42 & 1.71 & 1.53 & 1.71 & $6.94^{*}$ & $(.08)$ & 1.49 & $(.03)$ & 0.39 & $(.01)$ \\
\hline NACCS & 2.30 & 2.36 & 2.45 & 2.49 & 2.47 & 2.30 & 0.08 & $(.00)$ & 1.15 & $(.02)$ & 1.02 & $(.02)$ \\
\hline \multicolumn{13}{|c|}{ Measures from participants } \\
\hline \multicolumn{13}{|c|}{ Coherence } \\
\hline CES & 3.91 & 3.94 & 2.80 & 3.18 & 3.92 & 4.29 & 1.10 & $(.01)$ & $12.60^{* * * *}$ & $(.20)$ & 0.34 & $(.00)$ \\
\hline Life story & 5.87 & 5.20 & 4.53 & 4.67 & 5.93 & 6.07 & 0.14 & $(.00)$ & $7.65^{* *}$ & $(.14)$ & 0.80 & $(.01)$ \\
\hline Coherence & 4.89 & 4.68 & 4.82 & 4.52 & 5.18 & 4.68 & 1.45 & $(.02)$ & 0.43 & $(.01)$ & 0.14 & $(.00)$ \\
\hline Valence & 1.60 & 0.73 & 2.00 & 2.33 & -1.60 & -2.80 & $3.97^{*}$ & $(.02)$ & $117.41^{* * * *}$ & $(.68)$ & $3.65^{*}$ & $(.02)$ \\
\hline
\end{tabular}

Comprehension, disorganization, coherence and life story are all 1-7 scales; the CES is a 1-5 scale; and the NACCS is a $1-3$ scale; and valence is a -3 to +3 scale.

${ }^{*} p<.05$.

** $p<.01$.

${ }^{* * *} p<.001$.

**** $p<.0001$. 
tion of our narratives because they have been widely used in studying narratives of stressful and traumatic events. In addition, based on the literature (Pennebaker, Mehl, \& Niederhoffer, 2003) and the type of events used here, we report separately on positive and negative emotion words and on the cognitive subcategories of insight and cause words. We also report on the first person singular pronoun, $I$, because it has been linked to depression and suicide (Rude, Gortner, \& Pennebaker, 2004; Stirman \& Pennebaker, 2001).

\section{Results}

\subsection{Group and trauma characteristics}

There was no overlap in the PCL scores of the two groups. The PTSD group had higher scores on the PCL than the non-PTSD group (mean $=55.20, S D=5.77$, range of 46 to 65 versus mean $=24.87, S D=4.00$, range of $20-32, F(1,28)=279.98 p<.0001$ ). The PTSD group also had higher scores on the BDI-II (mean $=18.87, S D=8.46$ versus mean $=4.13, S D=2.83, F(1,28)=40.94$, $p<.0001$ ) and the DES (mean $=21.69, S D=12.56$ versus mean $=9.98, S D=6.07, F(1,28)=10.55, p<.01$ ).

The traumas described in the participants' narratives, which were the traumas on which the diagnoses were made, could be grouped into a small number of categories. The number of PTSD and non-PTSD participants in each category is listed after the category name: sexual assault, rape, or childhood sexual assault $(6,1)$; other serious crime $(3,0)$; suicide or attempted suicide of a parent, other family member, or friend $(2,3)$; other death of a parent, sibling, other family member, or friend $(1,6)$; sudden serious or life threatening accident or illness to participant, sibling, or parent $(3,5)$. The trauma categories are a broad range of traumatic events as defined by the DSM-IV-TR and are fairly well matched across the two groups. If there were a difference, it would be in the direction of somewhat more serious traumas in the PTSD group, which according to the prevalent view being challenged here would work in favor of their memories being more incoherent.

\subsection{Measures of coherence}

Tables 1 and 2 contain the means and ANOVAs for the key measures included in this study. In Table 1, we include six measures of coherence. We do not correct for multiple comparisons in the Tables or in our reporting because we are arguing against the main effect and interaction hypotheses and do not want to bias the results against finding them by raising the $p$ level.

Three of the six measures of narrative coherence show no statistically significant effects: comprehension, the Narrative Coherence Scheme, and the participants' own ratings of the coherence of their narratives. One of the six measures shows an effect only of having PTSD; people with PTSD are rated as having more disorganization for all three kinds of events, consistent with Gray and Lombardo (2001). But the means for this measure show that these memories are not disorganized; their mean rating in Table 1 range from 1.42 to 1.98, where the scale was anchored at 1 being none, 4 being half, and 7 being almost all of the text was disorganized. Moreover, with regard to the effects of PTSD symptoms, disphoria, and dissociation, the PCL, BDI, and DES did not correlate significantly with any of the measures of coherence of the traumatic memory shown in Table 1, though they correlated $-.62,-.55$, and -.43 with valence (all $p s<.05$ ). The remaining two measures show significant effects only for the type of event: the CES and life story, which both measure how well the event fits into the life of the individual and not the internal coherence of the memory of the event. Consistent with earlier work, these are most

Table 2

Measures from computer scoring of the narrative.

\begin{tabular}{|c|c|c|c|c|c|c|c|c|c|}
\hline \multirow[t]{3}{*}{ Measures } & \multicolumn{6}{|l|}{ Means } & \multicolumn{3}{|l|}{ ANOVAs } \\
\hline & \multicolumn{2}{|c|}{ Important PTSD } & \multicolumn{2}{|c|}{ Positive PTSD } & \multicolumn{2}{|c|}{ Traumatic PTSD } & \multirow{2}{*}{$\begin{array}{l}F(1,28) \\
\text { PTSD }\end{array}$} & \multirow{2}{*}{$\begin{array}{l}F(2,28) \\
\text { memory type }\end{array}$} & \multirow{2}{*}{$\begin{array}{l}F(2,28) \\
\text { interaction }\end{array}$} \\
\hline & No & Yes & No & Yes & No & Yes & & & \\
\hline \multicolumn{10}{|c|}{ LIWC categories (percent of words) } \\
\hline Emotional & 5.41 & 5.28 & 6.30 & 6.66 & 4.26 & 4.96 & 0.33 & $5.94^{* *}$ & 0.29 \\
\hline Positive & 3.95 & 3.09 & 5.33 & 5.40 & 1.80 & 2.12 & 0.17 & $21.19^{* * * * *}$ & 0.99 \\
\hline Negative & 1.44 & 2.21 & 1.07 & 1.26 & 2.42 & 2.84 & 1.17 & $6.58^{* *}$ & 0.27 \\
\hline Cognitive & 17.39 & 17.13 & 16.00 & 17.14 & 13.52 & 16.45 & 2.73 & $3.33^{*}$ & 1.56 \\
\hline Insight & 3.19 & 3.34 & 2.24 & 2.49 & 1.59 & 2.28 & 1.64 & $10.98^{* * * * *}$ & 0.48 \\
\hline Cause & 2.01 & 1.91 & 1.23 & 0.97 & 0.88 & 1.28 & 0.00 & $7.36^{* *}$ & 0.88 \\
\hline Sensory & 1.76 & 1.47 & 2.36 & 2.09 & 1.65 & 2.55 & 0.10 & $3.86^{*}$ & $4.29^{*}$ \\
\hline Social & 7.80 & 8.04 & 10.06 & 9.10 & 12.45 & 12.60 & 0.02 & $9.15^{* * *}$ & 0.19 \\
\hline I & 11.08 & 9.49 & 8.17 & 9.54 & 7.08 & 9.05 & 0.49 & $5.34^{* *}$ & $3.83^{*}$ \\
\hline Words/essay & 182.2 & 180.7 & 172.7 & 168.5 & 249.1 & 219.9 & 0.43 & $22.01^{* * * * *}$ & 1.06 \\
\hline Flesch & 66.22 & 66.96 & 69.13 & 70.64 & 73.29 & 75.16 & 0.26 & $6.12^{* *}$ & 0.03 \\
\hline
\end{tabular}

${ }^{*} p<.05$

** $p<.01$.

*** $p<.001$.

***** $p<.0001$ 
coherent for the traumatic event. Moreover, none of the coherence measures have means that would imply that the narratives are in the incoherent range - that is, disorganized enough to be a mechanism in the formation or maintenance of PTSD. Thus, there is no general tendency for participants with PTSD or for traumatic events to produce less coherent narratives. Moreover, there are no interactions of PTSD and event type for any of these six measures; thus, there is no support for the main effect and interaction hypotheses.

\subsection{Other measures}

The LIWC data is presented because it is used in many studies of memories of negative events (Pennebaker et al., 2003; Rude et al., 2004). Three observations are intriguing. First, the overall proportion of cognitive words, particularly cause and insight words, which are often mentioned as agents of change in expressive writing (Pennebaker, Mayne, \& Francis, 1997), is lowest in trauma narratives. Second, there is an interaction in the proportion of sensory words caused by participants with PTSD using a higher proportion of sensory words in their trauma narratives and a lower proportion in narratives of their important and positive memories. This is consistent with claims that trauma memories are richer in sensory details in people with PTSD (Ehlers \& Clark, 2000). Third, there is an interaction in the proportion of the first personal pronoun I caused by participants with PTSD using a higher proportion of the pronoun I in their trauma and positive memory narratives and a lower proportion in their important memory narratives. Thus, the relation between depression and increased use of the first personal pronoun I (Rude et al., 2004) may manifest itself in not just negative but in all emotional event narratives. The trauma memories are longer than the other memories and have higher Flesch Reading Scale values.

\section{Discussion}

Neither the existing literature, as reviewed earlier, nor our current results offer support for main effect the hypothesis that memories of traumatic events are more incoherent in general or for the interaction hypothesis that they are especially incoherent in people with PTSD. In this study, we measured the coherence of traumatic, very positive, and very important memories using self-reports from our participants' and judges' ratings of our participants' narratives. The lack of the main effect and interaction hypotheses is in spite of using a wide variety of measures of coherence and of having comparison events that should be especially coherent, making it easier for traumatic events to be less coherent by comparison.

Our findings that trauma memories are not incoherent compared to comparison memories and that there are no interactions of event type with PTSD support null hypotheses that we fail to reject. This paragraph provides reasons why our findings cannot be dismissed as a simple null hypothesis confirmation caused by insufficient power or a major flaw that prevented us from seeing results that were really present. First, the means of our various measures of coherence were not in a range that would be considered incoherent, which is how the main-effects and interaction hypotheses are formulated. Second, the means do not show a pattern that supports the claim that memories of traumatic events are more incoherent in general or in people with PTSD are more incoherent; that is, the trauma memories in general or in the PTSD group are not less coherent than the other memories, rather they tend to be more coherent. In terms of the main-effects hypothesis, trauma memories are the numerically the most coherent in four of the six measures and least coherent in none. In terms of the interaction hypothesis, trauma memories are the numerically the most coherent or tied for the most coherent in the PTSD participants in five of the six measures and least coherent in one. Third, effect sizes are generally small, and among those greater than .03 , only one supports the main-effects or interaction hypotheses. There are only four effect sizes greater than .03 in Table 1. One is for the manipulation check of valence $\left(\boldsymbol{\eta}^{2}=.68\right)$. There are two effects sizes in which the trauma memory is more coherent in the sense that it is more central to the identity $\left(\boldsymbol{\eta}^{2}=.20\right)$ or life story $\left(\boldsymbol{\eta}^{2}=.14\right)$, and one in which participants with PTSD produce trauma and control memories which are more disorganized $\left(\boldsymbol{\eta}^{2}=.08\right)$, though still showing little disorganization. Fourth, it would not take that much to produce the theoretically relevant interaction with the current data. If, for each measure, we were to set the means of the trauma memories to be equal to $20 \%$ less than the average means of the positive and important memories by subtracting the same constant from each participant in the PTSD and the nonPTSD group, then the main effect hypothesis would hold for all six measures of coherence (five at the .001 level). If we reduced only the values of the PTSD group by $20 \%$, the interaction would hold for four of the six measures. Given that the theoretically interesting claim is not about slightly less coherence of trauma memories in people with PTSD, but of incoherent memories, decreases of $20 \%$ in the coherence of such memories is a reasonable test.

One limitation of any study that waits the month required for a PTSD diagnosis is that memories are probably more confused right after a trauma, or after any other unusual event, until the person has time to reflect, and we do not measure this initial incoherence. The flashbulb memory literature indicates that people construct a relatively stable narrative in less than a week (Rubin, 1992). I know of no comparable estimate for traumas available; however, our measures are made at least 1 month after the trauma by which time many memories of trauma may have reached a relatively stable and more coherent version. Another limitation is that we, like other studies in the literature, did not record measures, which in hindsight would have been useful in interpreting the results. These include for all participants the lifetime history of traumas, the kinds of therapy that might have changed participant's memory of their trauma, a general history of retelling of the traumas and control events and whether participants ever had symptoms that might have allowed a diagnosis of PTSD for the traumatic event described or other events. 
The main advantage of restricting the age range and level of educational achievement of the participants is that it should reduce variability in our measures thereby making it easier to see any differences caused by having a diagnosis of PTSD. The main disadvantage is that other populations might produce different results. This is more likely because of the high level of cognitive functioning of the undergraduates who, to be recruited, had to be making progress in a competitive intellectual environment. This might afford them the reserve to hide deficiencies that might otherwise be evident in another population. The test for this concern would be to perform a similar study using other populations. Different results might be observed, but the existing literature reviewed earlier using other populations in incomplete versions of the design used in this study does not predict this outcome.

In summary, either from the literature in general or from this study, in which appropriate comparison groups and memories are included, there is no evidence that people with PTSD have more incoherent memories of their traumas.

\section{Acknowledgments}

I wish to thank Dorthe Berntsen, Adriel Boals, Joseph Fitzgerald, John Kihlstrom, and Ira Hymn for comments; Katie Barzee, Nicole Feeling, and Ivy Paw for their help in developing the scoring systems, Gustavo Araujo and Polly vande Velde for their help in recruiting and screening the participants, and NIMH Grant R01 MH066079 for support.

\section{References}

American Psychiatric Association (2000). Diagnostic and statistical manual of mental disorders (4th ed.). Washington, DC: American Psychiatric Association. Text Revision.

Amir, N., Stafford, J., Freshman, M. S., \& Foa, E. B. (1998). Relationship between trauma narratives and trauma pathology. Journal of Traumatic Stress, 11, 385-392.

Baker-Ward, L., Bauer, P. J., Fivush, R., Haden, C.A., Ornstein, P.A., \& Reese, E. (July, 2007). Coding coherence in autobiographical narratives. In Symposium conducted at the biennial meetings of the Society for Applied Research in Memory and Cognition, Lewiston, ME.

Beck, A. T., Steer, R. A., \& Brown, G. K. (1996). Manual for the beck depression inventory - II. San Antonio, TX: Psychological Corporation.

Berliner, L., Hyman, I. E., Jr., Thomas, A., \& Fitzgerald, M. (2003). Children's memory for trauma and positive experiences. Journal of Traumatic Stress, 16, 229-236.

Bernstein, E. M., \& Putnam, F. W. (1986). Development, reliability and validity of a dissociation scale. Journal of Nervous and Mental Disease, 174, 727-734.

Berntsen, D. (2009). Involuntary autobiographical memories. An introduction to the unbidden past. Cambridge University Press.

Berntsen, D., \& Rubin, D. C. (2006). The centrality of event scale: A measure of integrating a trauma into one's identity and its relation to post-traumatic stress disorder symptoms. Behaviour Research and Therapy, 44, 219-231.

Berntsen, D., \& Rubin, D. C. (2006). Flashbulb memories and posttraumatic stress reactions across the life-span: Age-related effects of the German occupation of Denmark during WWII. Psychology and Aging, 21, 127-139.

Berntsen, D., \& Rubin, D. C. (2007). When a trauma becomes a key to identity: Enhanced integration of trauma memories predicts posttraumatic stress disorder symptoms. Applied Cognitive Psychology, 21, 417-431.

Berntsen, D., \& Rubin, D. C. (2008). The reappearance hypothesis revisited: Recurrent involuntary memories after traumatic events and in everyday life. Memory E Cognition, 36, 449-460.

Berntsen, D., Rubin, D. C., \& Bohni, M. K. (2008). Contrasting models of posttraumatic stress disorder: Reply to Monroe and Mineka (2008). Psychological Review, 115, 1099-1106.

Berntsen, D., Willert, M., \& Rubin, D. C. (2003). Splintered memories or vivid landmarks? Qualities and organization of traumatic memories with and without PTSD. Applied Cognitive Psychology, 17, 675-693.

Blake, D. D., Weathers, F. W., Nagy, L. M., Kaloupek, D. G., Gusman, F. D., Charney, D. S., et al (1995). The development of a clinician-administered posttraumatic stress disorder scale. Journal of Traumatic Stress, 8, 75-80.

Brewin, C. R. (2001). Memory processes in post-traumatic stress disorder. International Review of Psychiatry, 13, $159-163$.

Brewin, C. R., Dalgleish, T., \& Joseph, S. (1996). A dual representation theory of posttraumatic stress disorder. Psychological Review, 103, 670-686.

Brewin, C. R., \& Holmes, E. A. (2003). Psychological theories of posttraumatic stress disorder. Clinical Psychology Review, $23,339-376$.

Byrne, C. A., Hyman, I. E., Jr., \& Scott, K. (2001). Comparisons of memories for traumatic events and other experiences. Applied Cognitive Psychology, 15, s119-s133.

Carlson, E. B., \& Putnam, F. W. (1993). An update on the dissociative experiences scale. Dissociation, 6, 16-27.

Dalgleish, T. (2004). Cognitive approaches to posttraumatic stress disorder: The evolution of multirepresentational theorizing. Psychological Bulletin, 130, $228-260$.

Ehlers, A., \& Clark, D. M. (2000). A cognitive model of posttraumatic stress disorder. Behaviour Research and Therapy, 38, $319-345$.

Flesch, R. (1948). A new readability yardstick. Journal of Applied Psychology, 32, 221-233.

Foa, E. B., Molnar, C., \& Cashman, L. (1995). Change in rape narratives during exposure therapy for posttraumatic stress disorder. Journal of Traumatic Stress, 8, 675-690.

Foa, E. B., Riggs, D. S., \& Gershuny, B. S. (1995). Arousal, numbing, and intrusion: Symptom structure of PTSD following assault. American Journal of Psychiatry, 152, 116-120.

Geraerts, E., Kozari-Kova, I. D., Merckelbach, H., Peraica, T., Jelicic, M., \& Candel, I. (2007). Traumatic memories of war veterans: Not so special after all. Consciousness and Cognition, 16, 170-177.

Gray, M. J., \& Lombardo, T. W. (2001). Complexity of trauma narratives as an index of fragmented memory in PTSD: A critical analysis. Applied Cognitive Psychology, 15, S171-S186.

Greenberg, D. L., \& Rubin, D. C. (2003). The neuropsychology of autobiographical memory. Cortex, 39, 687-728.

Halligan, S. L., Micheal, T., Clark, D. M., \& Ehlers, A. (2003). Posttraumatic stress disorder following Assault: The role of cognitive processing, trauma memory, and appraisals. Journal of Consulting and Clinical Psychology, 71, 419-431.

Harvey, A. G., \& Bryant, R. A. (1999). A qualitative investigation into the organization of traumatic memories. British Journal of Clinical Psychology, 38, 401-405.

Horowitz, M. J. (1976). Stress response syndromes. New York: Jason Aronson.

Jones, C., Harvey, A. G., \& Brewin, C. R. (2007). The organization and content of trauma memories in survivors of road traffic accidents. Behaviour Research and Therapy, 45, 151-162.

McNally, R. J. (2003a). Progress and controversy in the study of posttraumatic stress disorder. Annual Review of Psychology, 54, $229-252$.

McNally, R. J. (2003b). Remembering trauma. Cambridge: Harvard University Press.

Morris, S. G., Baker-Ward, L., \& Bauer, P. J. (2009). What remains of that day: The survival of children's autobiographical memories across time. Applied Cognitive Psychology, 23, 1-18. 
Murray, J., Ehlers, A., \& Mayou, R. A. (2002). Dissociation and post-traumatic stress disorder: Two prospective studies of road traffic accident survivors. British Journal of Psychiatry, 180, 363-368.

O'Kearney, R., \& Perrott, K. (2006). Trauma narratives in posttraumatic stress disorder: A review. Journal of Traumatic Stress, 19, 81-93.

Peace, K. A., Porter, S., \& ten Brinke, L. (2008). Are memories for sexually traumatic events "special"? A within-subjects investigation of trauma and memory in a clinical sample. Memory, 16, 10-21.

Pennebaker, J. W., Booth, R. E., \& Francis, M. E. (2007). Linguistic Inquiry and Word Count: LIWC2007-Operator's manual. Austin, TX: LIWC.net.

Pennebaker, J. W., Mayne, T., \& Francis, M. E. (1997). Linguistic predictors of adaptive bereavement. Journal of Personality and Social Psychology, 72, 863-871.

Pennebaker, J. W., Mehl, M. R., \& Niederhoffer, K. (2003). Psychological aspects of natural language use: Our words, our selves. Annual Review of Psychology, $54,547-577$.

Porter, S., \& Birt, A. R. (2001). Is traumatic memory special? A comparison of traumatic memory characteristics with memory for other emotional life experiences. Applied Cognitive Psychology, 15, 101-118.

Rubin, D. C. (1992). Constraints on memory. In E. Winograd \& U. Neisser (Eds.), Affect and accuracy in recall: Studies of "flashbulb" memories (pp. 265-273). New York: Cambridge University Press.

Rubin, D. C., Berntsen, D., \& Bohni, M. K. (2008). A memory-based model of posttraumatic stress disorder: Evaluating basic assumptions underlying the PTSD diagnosis. Psychological Review, 115, 985-1011.

Rubin, D. C., Boals, A., \& Berntsen, D. (2008). Memory in posttraumatic stress disorder: Properties of voluntary and involuntary, traumatic and non-traumatic autobiographical memories in people with and without PTSD symptoms. Journal of Experimental Psychology: General, 137, $591-614$.

Rubin, D. C., Boals, A., \& Klein, K. (2010). Autobiographical memories for very negative events: The effects of thinking about and rating memories. Cognitive Therapy and Research, 34, 35-48.

Rubin, D. C., Feldman, M. E., \& Beckham, J. C. (2003). Reliving, emotions, and fragmentation in the autobiographical memories of veterans diagnosed with PTSD. Applied Cognitive Psychology, 18, 17-35.

Rubin, D. C., Schrauf, R. W., \& Greenberg, D. L. (2003). Belief and recollection of autobiographical memories. Memory E Cognition, 31, 887-901.

Rubin, D. C., Schrauf, R. W., \& Greenberg, D. L. (2004). Stability in autobiographical memories. Memory, 12, 715-721.

Rude, S. S., Gortner, E. M., \& Pennebaker, J. W. (2004). Language use of depressed and depression-vulnerable college students. Cognition and Emotion, 18, $1121-1133$.

Shobe, K. K., \& Kihlstrom, J. F. (1997). Is traumatic memory special? Current Directions in Psychological Science, 3, 70-74.

Stirman, S. W., \& Pennebaker, J. W. (2001). Word use in the poetry of suicidal and non-suicidal poets. Psychosomatic Medicine, 63, $517-522$.

Van der Kolk, B. A., \& Fisler, R. (1995). Dissociation and the fragmentary nature of traumatic memories: Overview and exploratory study. Journal of Traumatic Stress, 8, 505-525.

Weathers, F. W., Litz, B. T., Huska, J. A., \& Keane, T. M. (1994). The PTSD Checklist (PCL). Unpublished scale available from the National Center for PTSD.

Zoellner, L. A., \& Bittenger, J. N. (2004). On the uniqueness of trauma memories in PTSD. In G. M. Rosen (Ed.), Posttraumatic stress disorder: Issues and controversies (pp. 147-162). Chichester, England: John wiley \& Sons, Ltd.. 